\title{
ATM induces radioresistance of non-small cell lung cancer A549 cells by downregulation of MDMX
}

\author{
R. Xing1, J.J. Chen', M.Y. Chen'1, J. Lian'1, L.F. Li'1, X. Zhou'1, R.Q. Liu'1, \\ Y.Z. Xie ${ }^{1}$, W. Huang ${ }^{1}$ H. Zhao', Y.C. Zeng, ${ }^{1,2 *}$ \\ ${ }^{1}$ Department of Clinical Oncology, Shengjing Hospital of China Medical University, Shenyang, China \\ ${ }^{2}$ Department of Medical Oncology, Cancer Center, The Second Affiliated Hospital of Hainan Medical University, \\ Haikou, China.
}

\section{- Original article}

\author{
*Corresponding authors: \\ Yue-Can Zeng, M.D., Ph.D., \\ E-mail: \\ wellyy2005@hotmail.com \\ Revised: February 2020 \\ Accepted: March 2020 \\ Int. J. Radiat. Res., October 2020; \\ 18(4): $835-840$ \\ DOI: 10.18869 /acadpub.ijrr.18.4.835
}

\begin{abstract}
Background: Tumor radioresistance leads to a reduction in the efficiency of radiation therapy. It is very important to explore the cellular mechanisms leading to radioresistance and to find potential therapeutic targets, which might improve the efficacy of radiation therapy. This study was to investigate the role of ataxia-telangiectasia mutated (ATM) and murine double minute $X$ (MDMX) in radioresistance in non-small cell lung cancer A549 cells and their corresponding mechanisms of action. Materials and Methods: Non-small cell lung cancer A549 cells were irradiated with X-rays in the presence or absence of ATM inhibitor. Cell survival, cell apoptosis, cell proliferation, mRNA of ATM and MDMX, and protein expression of ATM, MDMX, $\gamma-\mathrm{H} 2 \mathrm{AX}$, Caspase3, and Beclin1 were measured. Results: After the inhibitor (KU60019) treatment combined with $\mathrm{X}$ irradiation, the $\mathrm{A} 549$ cells showed a significant decrease in colony formations compared to the group received irradiation alone. The MDMX knockdown A549 cells showed a significant increase in colony formations compared to the control group. ATM downregulated the expression of MDMX after irradiation treatment in A549 cells. Irradiation led to a significant increase in $\mathrm{Y}-\mathrm{H} 2 \mathrm{AX}$ expression, but MDMX knockdown decreased the $\mathrm{\gamma}-\mathrm{H} 2 \mathrm{AX}$ expression after irradiation. The change of Caspase 3 expression was the same as $\mathrm{\gamma}-\mathrm{H} 2 \mathrm{AX}$. Irradiation led to a significant increase of Beclin1 expression and MDMX knockdown increased the Beclin1 expression after irradiation. Conclusion: This study indicated that ATM induced radioresistance through downregulating the expression of MDMX, which was at least partly associated with the activation of autophagy and the decrease of DNA damage in A549 cells.
\end{abstract}

Keywords: ATM, MDMX, radioresistance, non-small cell lung cancer.

\section{INTRODUCTION}

Radiotherapy is one of the most widely used management modalities for non-small cell lung cancer (NSCLC) (1). Unfortunately, the treatment outcomes in some patients are not satisfactory. Tumor radioresistance leads to a reduction in the efficiency of radiation therapy with corresponding tumor metastasis and/or recurrence $(2,3)$. Therefore, it is very important to study the cellular mechanisms leading to radioresistance and to discover potential therapeutic targets, which might improve the efficacy of radiation therapy (4).

Ataxia-telangiectasia mutated (ATM), a serine/threonine-protein kinase, functions as a transducer of the DNA damage signal to the downstream molecules involved in the Homologous recombination repair (HRR)mediated double-strand breaks (DSBs) repair pathway ${ }^{(5)}$. ATM hyperphosphorylation activates the downstream molecules, such as p53 and murine double minute 2(MDM2), etc., which leads to cell cycle arrest.[6] The important role of 
ATM in the response to radiation is strengthened by ATM-deficient human cell lines (A-T cells), which are sensitive to ionizing radiation (7).In other words, ATM-wild/rich cells may display radioresistance (8). Murine double minute $\mathrm{X}$ (MDMX) resembles MDM2 at the $\mathrm{N}$-terminal p53 -binding and the $\mathrm{C}$-terminal ring finger domains. Like MDM2, MDMX binds to p53 and inhibits its functions ${ }^{(9)}$. Recent findings suggest that MDM2 and MDMX are specific independent therapeutic targets for activating wild-type p53. Thus, anti-cancer approaches that target both MDM2 and MDMX should be considered as a means of cancer treatments (10).

As both ATM and MDMX functioned through regulation of p53, we hypothesized that there may be some relationships between ATM and MDMX in producing radioresistance in NSCLC (11). However, there are no reported studies on investigating the relationships between ATM and MDMX in NSCLC. Thus, to explore the mechanisms of ATM modulating the radioresistance through MDMX in NSCLC is novel and very important. This preliminary study aimed to investigate the mechanisms of ATM regulating MDMX in inducing radioresistance in non-small cell lung cancer A549 cells.

\section{MATERIALS AND METHODS}

\section{Cell culture and reagents}

Non-small cell lung cancer line A549 (obtained from American Type Culture Collection, ATCC) was cultured in RPMI 1640 medium(Gibco, Grand Island, NY, USA) under conditions of $5 \% \mathrm{CO} 2$ in an incubator (LWSE-350, Hanzhou, China) at $37^{\circ} \mathrm{C}$. KU60019 was purchased from Sigma-Aldrich Co. and was dissolved in 100\% DMSO (dimethyl sulfoxide, Sigma-Aldrich Co.) and stored at $-20^{\circ} \mathrm{C}$.

\section{Irradiation of cells}

Cells were exposed to $6 \mathrm{MV} \mathrm{X}$ radiation by using Elekta Synergy Linear Accelerator.

Different radiation doses (3Gy/min) was applied to all groups. After irradiation, the culture medium was changed immediately.

\section{Clonogenic formation assay}

A549 cells were seeded onto six-well dishes (Thermo Fisher Scientific Inc, USA). After overnight culture, the cells were treated with KU60019 $(5 \mu \mathrm{mol} / \mathrm{L}){ }^{(12)}$ or control for $24 \mathrm{~h}$. KU60019 is the ATM inhibitor and the experiment dose was referred to Tang's study (12). Cells were then irradiated at a dose of $0,2,4$, 6, and 8 Gy with $6-\mathrm{MV} \mathrm{X}$-rays, 3.0Gy/min. The cells were then cultured in a $5 \% \mathrm{CO}_{2}$ incubator at $37{ }^{\circ} \mathrm{C}$ for 7 days. The colonies were fixed and stained with crystal violet (Sigma-Aldrich Co. USA) for counting the number of colonies. The number of colonies containing at least 50 cells was determined, and the surviving fractions were calculated.

\section{Flow cytometric analysis of apoptosis}

A549 cells were irradiated at a single dose of 4 Gy after treatment with Ku60019 ( $5 \mu \mathrm{mol} / \mathrm{L})$ or control for $24 \mathrm{~h}$. Apoptosis of cells was detected by flow cytometry (BD FACSCalibur, USA). $5 \mu \mathrm{L}$ Annexin V/FITC (Abcam, USA) and $10 \mu \mathrm{L} 20 \mu \mathrm{g} /$ $\mathrm{mL}$ propidium iodide (PI, Abcam, USA) were added to the tube.

\section{Quantitative RT-PCR}

Total RNA was extracted using TRIZOL reagent (Invitrogen, USA) from non-small lung cancer cell pellets according to the reagent instructions. An equal amount of RNA $(10 \mu \mathrm{g})$ was reversely transcribed into cDNA by reverse transcriptase according to the protocol. ATM, MDMX and GAPDH (glyceraldehyde 3-phosphate dehydrogenase) were then amplified by quantitative real-time PCR by using the following primers: ATM: forward: 5'-AAC ATA CTA CTC AAA GAC ATT CCT GTC TC-3', reverse: $5^{\prime}$-AAA ATG TCT TTG AGT AG T ATG CCT GTC TC-3'; MDMX: forward: $5^{\prime}$-CAGCAGGTGCGCAAGGTGAA3', reverse: 5'-CTGTGCGAGAGCGAGAGTCTG-3'; GAPDH: forward: 5'-TGAAGGTCGGAGTCAACGG3', reverse: 5 -CTGGAAGATGGTGATGGGATT-3'. These specific primers were designed and synthesized by Shanghai Jikai Gene Chemical Technology Co., Ltd (Shanghai, China). Gene amplification was performed in a real-time PCR system (Thermo Fisher Scientific Inc, USA) by using the SYBR Green master mix (Thermo Int. J. Radiat. Res., Vol. 18 No. 4, October 2020 
Fisher Scientific Inc, USA). The mixture was preheated for $10 \mathrm{~min}$ at $95^{\circ} \mathrm{Cand}$ followed by 50 cycles of amplification $\left(30 \mathrm{~s}\right.$ at $95^{\circ} \mathrm{C}$ and $1 \mathrm{~min}$ at $58^{\circ} \mathrm{C}$, respectively). The $\mathrm{C}_{t}$ value of each sample was calculated, and the relative mRNA expressions of ATM and MDMX were normalized to the GAPDH ( $\mathrm{C}_{\mathrm{t}}$ method).

\section{Western blot analysis}

Western blot was performed according to the protocol. The cell proteins were separated by SDS-PAGE (sodium dodecyl sulfate polyacrylamide gel electrophoresis, SigmaAldrich Co., USA) and then transferred onto nitrocellulose membranes (Sigma-Aldrich Co., USA). The membranes were incubated with primary antibodies at $4^{\circ} \mathrm{C}$ overnight, including monoclonal antibodies against GAPDH (dilution 1:4000, Santa Cruz Biotechnology, USA), monoclonal antibodies against ATM, MDMX, $\gamma-\mathrm{H} 2 \mathrm{AX}$, Caspase 3 and Beclin1 (dilution 1:100, Santa Cruz Biotechnology, USA). The membranes were incubated at room temperature for $2 \mathrm{~h}$ with HRP(horseradish peroxidase)-conjugated goat anti-mouse or goat anti-rabbit IgG antibodies (Sigma-Aldrich Co., USA) after three washes with TBST (Tris buffered saline with Tween-20, Sigma-Aldrich Co., USA). Molecular Images ChemiDoc XRS+ imager with image lab software (Bio-Rad Laboratories, Inc., USA) was applied to visualize the intensity of protein bands.

\section{Transfection}

The expression of MDMX was down-regulated by cloning MDMX-shRNA cassette in the PTRIPZ lentiviral system (Applied Biosystems, USA). The viral particles were generated by co-transfecting the recombinant MDMX-shRNA-pTRIPZ constructs and packaging plasmids in HEK293FT cells. Further, Non-small cell lung cancer line A549 was transduced. The stable selection was performed by using a puromycin selection marker. The expression for shRNA was induced after $72 \mathrm{~h}$ of treatment by doxycycline, the protein levels of MDMX were evaluated by western blot. A non-targeting oligonucleotide sequence was cloned as the control group.

Int. J. Radiat. Res., Vol. 18 No. 4, October 2020

\section{Soft agar assay}

The soft agar assay was done according to the protocol. Cancer cells were mixed with $0.3 \%$ agarose (Sigma-Aldrich Co., USA) and plated onto a $0.5 \%$ agarose underlay $\left(1 \times 10^{4}\right.$ cells $/$ well in six-well plates). The number of foci $>100 \mu \mathrm{m}$ was calculated after 2 weeks.

\section{Statistical analysis}

All statistical analyses were performed using SPSS19.0 (IBM Corp., Armonk, NY, USA) and GraphPad Prism version 5 (GraphPad Software, Inc., USA). Statistical significance was determined with the unpaired Student's t-test. $\mathrm{P}<0.05$ was considered statistically significant.

\section{RESULTS}

\section{ATM inhibitor and MDMX affecting the radioresistance of $A 549$ cells}

The cells exhibited a decrease in their ability to forming colonies after radiation. After the ATM inhibitor treatment (KU60019) combined with X irradiation, the A549 cells showed a significant decrease in colony formations compared to the group received irradiation alone (figure 1A). The shMDMX (MDMX knockdown) A549 cells showed a significant increase in colony formations compared to the control group (figure 1B).

\section{MDMX downregulation by ATM}

As shown in figure 2, ATM downregulated the expression of MDMX after irradiation treatment in A549 cells, but the reduction could be prevented when treated with the ATM inhibitor (KU60019) combined with X irradiation.

\section{MDMX affecting the apoptosis and cell proliferation}

The flow cytometric analysis showed that MDMX knockdown significantly inhibited the apoptosis of A549 cells after irradiation (figure 3A, 3B). Irradiation decreased the colony number of A549 cells. MDMX knockdown plus irradiation significantly increased the colonies as compared to the irradiation group (figure 3C, 3D). 


\section{Western blot analysis of the effects of shMDMX on $A 549$ cells}

In this study, we performed western blot to explore the relationship between MDMX and some other downstream proteins in inducing radioresistance in $\mathrm{A} 549$ cells.

As shown in figure 4, irradiation downregulated the expression of MDMX. MDMX knockdown led to the most reduction of MDMX protein expression in A549 cells. Irradiation led to a significant increase in $\gamma$-H2AX expression, but MDMX knockdown decreased the $\gamma$-H2AX expression after irradiation in A549 cells, which indicated that MDMX knockdown decreased the radiation induced DNA damage. The change of Caspase 3 expression was the same as $\gamma-\mathrm{H} 2 \mathrm{AX}$, which indicated that MDMX knockdown decreased the radiation induced apoptosis. Irradiation led to a significant increase of Beclin1 expression. MDMX knockdown increased the Beclin1 expression after irradiation, which indicated that MDMX knockdown might increase autophagy after irradiation in A549 cells.
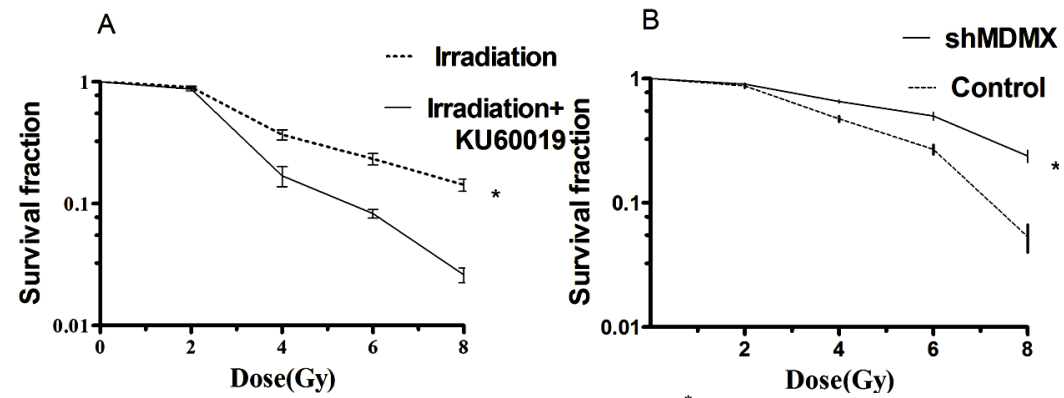

Figure 1. Cell survival fraction versus irradiation dose for $A 549$ cells. ${ }^{*} \mathrm{P}<0.05$, compared between these two groups.
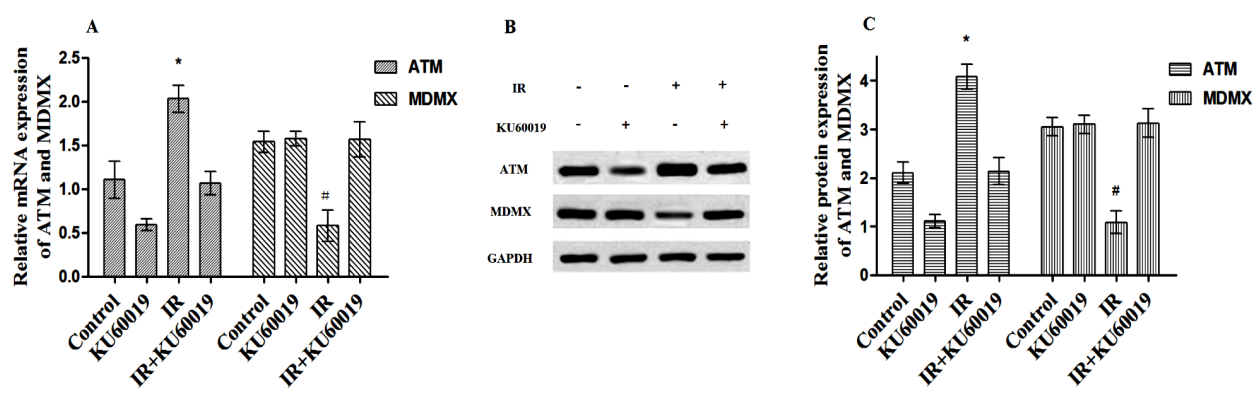

Figure 2. ATM and MDMX expression in A549 cells. (A) Irradiation increased the mRNA expression of ATM compared to the control group, but the inhibitor(KU00619) treatment combined with irradiation significantly inhibited the increase of ATM mRAN expression $\left({ }^{*} \mathrm{P}<0.05\right.$, compared to other groups); on the other hand, the change of MDMX mRNA expression was opposite to ATM

(\#P<0.05,compared to IR+KU00619 group). (B, C) Expressions of ATM and MDMX protein changed the same as the mRNA

$A$
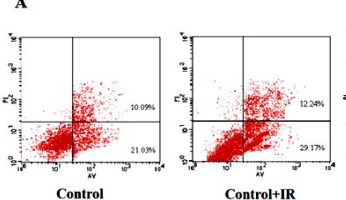

Control+IR

C

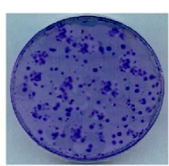

Control

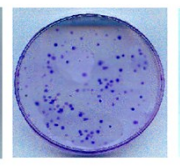

Control+IR
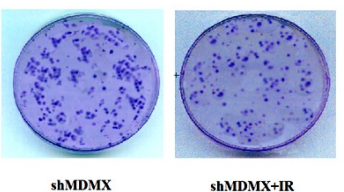

shMDMX+1R

B

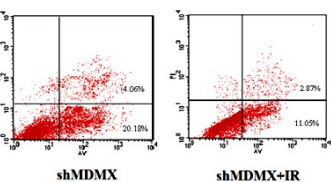

shMDMX+IR expression.
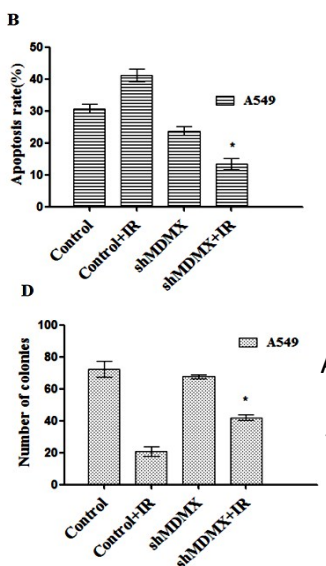

Int. J. Radiat. Res., Vol. 18 No. 4, October 2020
Figure 3. The effects of MDMX on apoptosis and cell proliferation. (A, B) Flow cytometric analysis showed that MDMX knockdown inhibited apoptosis of $A 549$ cells after irradiation $\left({ }^{*} P<0.05\right.$, compared to the irradiation group). (C, D) Soft agar assay showed that irradiation could successfully reduce the number of colonies in A549 cells. The combination of MDMX knockdown and irradiation together significantly increased the colonies as compared to the irradiation group $\left({ }^{*} \mathrm{P}<0.05\right)$. 


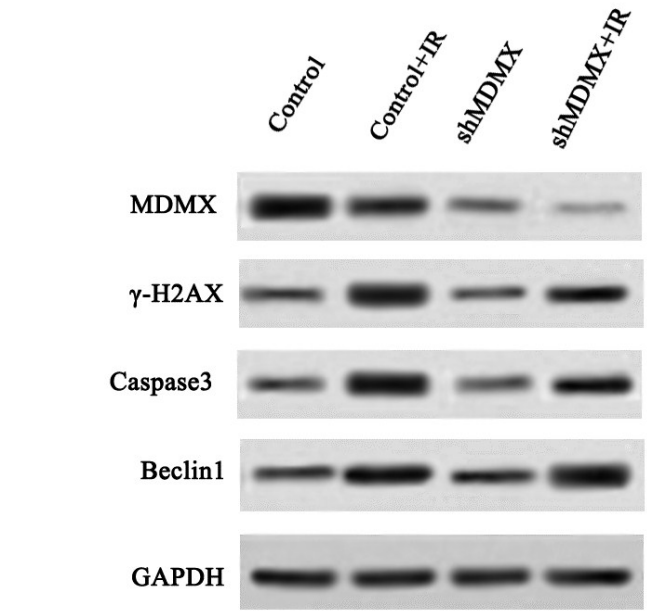

Figure 4. MDMX, $y-\mathrm{H} 2 \mathrm{AX}$, Caspase 3 and Beclin1 protein expression in $\mathrm{A} 549$ cells.

\section{DISCUSSION}

Lung cancer is the leading cause of cancer death worldwide. Non-small cell lung cancer accounts for approximately $80 \%$ of all lung cancers. About forty percent of all cases present with advanced stages, and many of them will be considered inoperable (13). Radiation therapy is recommended for the treatment of those patients in the palliative setting and for definitive management of unresectable nonmetastatic disease either alone or with concurrent chemotherapy (1). Long term exposure to irradiation usually induces an adaptive response in the cancer cells, which results in tolerance to subsequent radiation treatment with the generation of radioresistant residual cells that increase the risk of tumor recurrence, metastasis and negatively prejudices survival outcomes (14). Therefore, it is of vital importance to eradicate these radioresistant residual cells.

ATM, which is a sensor of DNA damage, plays a key role in the phosphorylation of p53 and DNA-PKcs ${ }^{(15)}$, both of which are vital regulators in the repair of irradiation-induced DNA DSBs. ATM kinase inhibition sensitized cells to irradiation(16). Because of its similarity with Mdm2 and its ability to inhibit p53-induced transcription after overexpression, MDMX was hypothesized to act as a novel negative regulator of p53 (17). There were reports indicated that both MDM2 and MDMX are required to inhibit p53 activity in the same cell type, which confirmed the notion that MDM2 can not compensate for MDMX loss(17). In our study, we investigated the relationship between ATM and MDMX in inducing radioresistance in non-small cell lung cells. As shown in Figure 1, MDMX knockdown increased the colony formation compared to the control group after irradiation. Previous reports showed that MDMX overexpression inhibits oncogene-induced senescence program and MDMX can regulate FL118 (a camptothecin analogue)-induced cell killing through the p53 pathway(18). Inhibiting the negative regulators MDMX and MDM2 stabilizes p53 and can potentiate radiotherapy outcomes (19). These results suggested that MDMX plays a role in the process of radiation induced DNA repair. To further investigate the relationship between ATM and MDMX in A549 cells after irradiation, we examined ATM and MDMX expression by RT-PCR and western blot. The results showed that ATM induced radioresistance through downregulating the expression of MDMX (figure 2) and ATM modified MDMX by affecting the cell apoptosis and cell proliferation (figure 3 ). It could be concluded that ATM induced radioresistance at least partly through downregulating the expression of MDMX.

Subsequent biological experiments indicated that MDMX knockdown significantly inhibited the apoptosis of A549 cells and increased the colonies as compared to the irradiation group. These results further confirmed that MDMX involved in the radioresistance in A549 cells after irradiation. To evaluate the possible mechanism by which MDMX promotes radioresistance, the expression levels of $\gamma$-H2AX, Caspase 3 and Beclin1 were detected by western blot. It is known that $\gamma-\mathrm{H} 2 \mathrm{AX}$ is a surrogate marker of radiation induced DSBs and is one of the most sensitive and selective biomarkers of DNA damage (20). Caspase3 serves as an executioner of apoptosis and mainly contributes to cell apoptosis. Besides apoptosis, it may affect the efficiency of current cancer treatment through promoting cell proliferation in chemo- or radiotherapy resistant cancer cells (21). Beclin1 is an important regulator of autophagy 
activation and has a key role in autophagy. The Caspase-mediated cleavage of Beclin1 can promote crosstalk between apoptosis and autophagy. Beclin 1 and Caspase involved in cross-regulations between apoptosis and autophagy ${ }^{(22) .}$

\section{CONCLUSION}

Taken together, our study indicated that ATM induced radioresistance through downregulating the expression of MDMX, which was at least partly associated with the activation of autophagy and the decrease of DNA damage in non-small cell lung cancer A549 cells. However, for it was a preliminary report, further studies on these two mechanisms by which ATM and MDMX are involved in the generation of radioresistance in non-small cell lung cancer and other cancer types are required.

\section{Funding sources}

This work was supported by the Foundation of Educational Commission of Liaoning Province of China (No. LQNK201714), the Key Projects of Department of Science and Technology of Liaoning Province (No. 2020JH2/10300142), the Doctoral Start-up Foundation of Liaoning Province (No. 20170520052), the Excellent Young Teachers Program of China Medical University (No. QGZ2018060) and the 345 Talent Project of Shengjing Hospital of China Medical University.

\section{Conflicts of interest: Declared none.}

\section{REFERENCES}

1. Reck M, Heigener DF, Mok T, Soria JC, Rabe KF(2013) Management of non-small-cell lung cancer: recent developments. Lancet, 382:709-19.

2. Salama JK and Vokes EE (2013) New radiotherapy and chemoradiotherapy approaches for non-small-cell lung cancer. J Clin Oncol, 31: 1029-38.

3. Bollineni VR, Wiegman EM, Pruim J, Groen HJ, Langendijk JA(2012) Hypoxia imaging using Positron Emission Tomography in non-small cell lung cancer: implications for radiotherapy. Cancer Treat Rev, 38: 1027-32.

4. Provencio M and Sánchez A (2014) Therapeutic integration of new molecule-targeted therapies with radiotherapy in lung cancer. Transl Lung Cancer Res, 3: 89-94.
5. Weber AM and Ryan AJ (2015) ATM and ATR as therapeutic targets in cancer. Pharmacol Ther, 149: 124-38.

6. Meulmeester E, Pereg Y, Shiloh Y, Jochemsen AG (2005) ATM-mediated phosphorylations inhibit Mdmx/ Mdm2 stabilization by HAUSP in favor of p53 activation. Cell Cycle, 4: 1166-70.

7. Tichý A, Vávrová J, Pejchal J, Rezácová M(2010) Ataxiatelangiectasia mutated kinase (ATM) as a central regulator of radiation-induced DNA damage response. Acta Medica (Hradec Kralove), 53: 13-7.

8. Tang FR and Loke WK (2015) Molecular mechanisms of low dose ionizing radiation-induced hormesis, adaptive responses, radioresistance, bystander effects, and genomic instability. Int J Radiat Biol, 91: 13-27.

9. Bista $M$, Petrovich $M$, Fersht AR (2013) MDMX contains an autoinhibitory sequence element. Proc Natl Acad Sci USA, 110: 17814-9.

10. Toledo $\mathrm{F}$ and Wahl GM (2007) MDM2 and MDM4: p53 regulators as targets in anticancer therapy. Int J Biochem Cell Biol, 39: 1476-82.

11. Cheng $Q$ and Chen J (2010) Mechanism of p53 stabilization by ATM after DNA damage. Cell Cycle, 9: 472-8.

12. Yuan $Z$, Guo $W$, Yang J, Li L, Wang $M$, Lei $Y$, Wan $Y$, Zhao $X$, Luo $N$, Cheng $P$, Liu $X$, Nie $C$, Peng $Y$, Tong A, Wei $Y$ (2015) PNAS-4, an early DNA damage response gene, induces $S$ phase arre st and apoptosis by activating checkpoint kinases in lung cancer cells. J Biol Chem, 290: 1492744.

13. De Santis $C E$, Lin $C C$, Mariotto $A B$, Siegel RL, Stein $\mathrm{KD}$, Kramer JL, Alteri R, Robbins AS, Jemal A (2014) Cancer treatment and survivorship statistics, 2014. CA Cancer J Clin, 64: 252-71.

14. Schuurbiers OC, Kaanders JH, van der Heijden HF, Dekhuijzen RP, Oyen WJ, Bussink J (2009) The PI3-K/ AKT-pathway and radiation resistance mechanisms in nonsmall cell lung cancer. J Thorac Oncol, 4: 761-7.

15. Knittel G, Liedgens P, Reinhardt HC (2015) Targeting ATMdeficient CLL through interference with DNA repair pathways. Front Genet, 6: 207.

16. Sarkaria JN, Busby EC, Tibbetts RS, Roos P, Taya Y, Karnitz LM, Abraham RT(1999) Inhibition of ATM and ATR kinase activities by the radiosensitizing agent, caffeine. Cancer Res, 59: 437582.

17. Marine JC, Francoz $S$, Maetens $M$, Wahl G, Toledo F, Lozano G (2006) Keeping p53 in check: essential and synergistic functions of $\mathrm{Md}$ $\mathrm{m} 2$ and Mdm4. Cell Death Differ, 13: 927-34.

18. Ling X, Xu C, Fan C, Zhong K, Li F, Wang X (2014) FL118 induces p53-dependent senescence in colorectal cancer cells by promoting degradation of MdmX. Cancer Res, $\mathbf{7 4 :}$ 7487-97.

19. Spiegelberg D, Mortensen AC, Lundsten S, Brown CJ, Lane DP, Nestor M (2018) The MDM2/MDMX-p53 Antagonist PM2 Radiosensitizes Wild-Type p53 Tumors. Cancer Res, 78: 5084-5093.

20. Solier S and Pommier Y (2014) The Nuclear $y$-H2AX Apoptotic Ring: Implications for Cancers and Autoimmune Diseases. Cell Mol Life Sci, 71: 2289-97.

21. Boland K, Flanagan L, Prehn JH (2013) Paracrine control of tissue regeneration and cell proliferation by Caspase-3. Cell Death Dis, 4: e725.

22. Kang R, Zeh HJ, Lotze MT, Tang D (2011) The Beclin 1 network regulates autophagy and apoptosis. Cell Death Differ, 18: 571-80.

Int. J. Radiat. Res., Vol. 18 No. 4, October 2020 